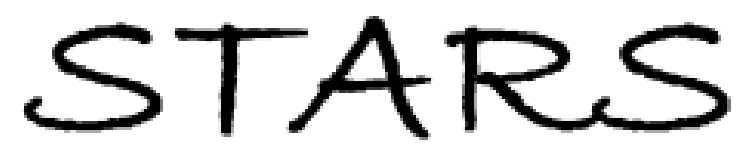

University of Central Florida

STARS

$1-1-2010$

\title{
Anomalous lattice dynamics and thermal properties of supported size- and shape-selected Pt nanoparticles
}

\author{
B. Roldan Cuenya \\ University of Central Florida
}
A. I. Frenkel
S. Mostafa
University of Central Florida
F. Behafarid
University of Central Florida
J. R. Croy
University of Central Florida

Find similar works at: https://stars.library.ucf.edu/facultybib2010

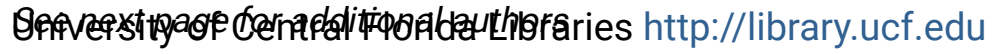

This Article is brought to you for free and open access by the Faculty Bibliography at STARS. It has been accepted for inclusion in Faculty Bibliography 2010 s by an authorized administrator of STARS. For more information, please contactSTARS@ucf.edu.

\section{Recommended Citation}

Cuenya, B. Roldan; Frenkel, A. I.; Mostafa, S.; Behafarid, F.; Croy, J. R.; Ono, L. K.; and Wang, Q., "Anomalous lattice dynamics and thermal properties of supported size- and shape-selected Pt nanoparticles" (2010). Faculty Bibliography 2010s. 74.

https://stars.library.ucf.edu/facultybib2010/74

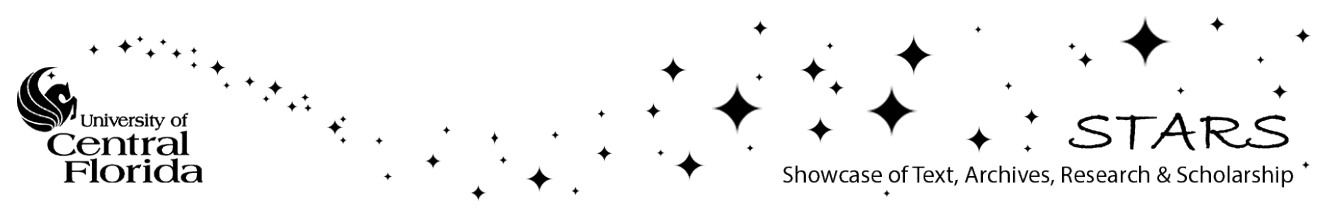


Authors

B. Roldan Cuenya, A. I. Frenkel, S. Mostafa, F. Behafarid, J. R. Croy, L. K. Ono, and Q. Wang 


\title{
Anomalous lattice dynamics and thermal properties of supported size- and shape-selected Pt nanoparticles
}

\author{
B. Roldan Cuenya, ${ }^{1, *, \dagger}$ A. I. Frenkel, ${ }^{2, *,+}$ S. Mostafa, ${ }^{1}$ F. Behafarid,${ }^{1}$ J. R. Croy,${ }^{1}$ L. K. Ono, ${ }^{1}$ and Q. Wang ${ }^{2}$ \\ ${ }^{1}$ Department of Physics, University of Central Florida, Orlando, Florida 32816, USA \\ ${ }^{2}$ Department of Physics, Yeshiva University, New York, New York 10016, USA
}

(Received 26 August 2010; published 28 October 2010)

\begin{abstract}
Anomalous lattice dynamics and thermal behavior have been observed for ligand-free, size-, and shapeselected Pt nanoparticles (NPs) supported on nanocrystalline $\gamma-\mathrm{Al}_{2} \mathrm{O}_{3}$ via extended x-ray absorption finestructure spectroscopy. Several major differences were observed for the NPs with respect to bulk Pt: (i) a contraction in the interatomic distances, (ii) a reduction in the dynamic (temperature-dependent) bond-length disorder and associated increase in the Debye temperature $\left(\theta_{D}\right)$, and (iii) an overall decrease in the bond-length expansion coefficient coupled with NP stiffening. The increase in the Debye temperature is explained in terms of the NP size, shape, support interactions, and adsorbate effects. For a similar average size, we observe a striking correlation between the shapes of the NPs and their $\theta_{D}$ values.
\end{abstract}

DOI: $10.1103 /$ PhysRevB.82.155450

PACS number(s): 61.05.cj, 61.46.Df, 65.80.-g, 82.60.Qr

\section{INTRODUCTION}

Metal nanoparticles (NPs) display anomalous vibrational properties such as phonon confinement effects and strong modifications of the phonon density of states as compared to bulk materials. ${ }^{1-6}$ Although some of the factors contributing to this unusual behavior are understood (e.g., NP interaction with the support, encapsulating ligands, and/or adsorbates), they are normally discussed in isolation. Furthermore, while it is recognized that environmental effects do contribute to the thermodynamics of small systems, ${ }^{7}$ very little is known on how to sort out and control such complex interactions at the nanoscale. The resolution of these challenges requires the combination of highly reproducible NP synthesis and sensitive methods for determining thermodynamic properties in different environments.

Recent extended x-ray absorption fine-structure (EXAFS) experiments revealed two peculiarities in the thermal properties of certain supported metal NPs: (i) a contraction in their nearest-neighbor (NN) bond lengths at elevated temperatures and (ii) an increase in their Einstein (and, hence, Debye, $\theta_{D}$ ) temperature with respect to bulk. ${ }^{8,9}$ The former effects were tentatively attributed to thermally induced dynamic changes in the structure of the NPs and to the strong interaction of the NPs with defects (vacancies) in the support. ${ }^{10} \mathrm{An}$ increased $\theta_{D}$ relative to the bulk has been reported for a variety of supported NP systems $\mathrm{s}^{8,9,11-13}$ and unsupported nanocrystalline agglomerates. ${ }^{14-16}$ By contrast, reduced values of $\theta_{D}$ have also been observed for seemingly analogous systems. ${ }^{17,18}$ The wide discrepancy observed for $\theta_{D}$ suggests that many factors are at play. In free clusters, enhanced surface energy and the concomitant increase in the surface stress causes a lattice contraction at the NP surface, softening of interatomic force constants, and a suppression of $\theta_{D}$ and melting temperatures. ${ }^{1}$ On the other hand, the increased $\theta_{D}$ observed for certain systems has been attributed to structural stiffening and inhomogeneous internal stress correlated with the NP size, surroundings (e.g., adsorbates/ surface ligands, ${ }^{15,16,19}$ a matrix encapsulating the NPs or a support $^{12,13}$ ), and to the presence of structural defects and multiple grains in large NPs. ${ }^{14}$ It is evident that the understanding of thermal, structural, and electronic properties of these systems is hindered by their complexity, due to the multiple competing factors that define their behavior, and any effort in sorting out these influences will be helpful for developing first-principles theories. In the present study we investigate the relationship between thermodynamic quantities $\left(\theta_{D}\right.$ and thermal-expansion coefficient) and geometrical properties of ligand-free, homogeneous, size-, and shapeselected Pt NPs supported on $\gamma-\mathrm{Al}_{2} \mathrm{O}_{3}$ using EXAFS.

\section{EXPERIMENTAL AND THEORETICAL METHODS}

\section{A. Sample preparation}

Size- and shape-selected Pt NPs were prepared by inverse micelle encapsulation methods using poly(styrene)-blockpoly(2vinylpyridine) (PS-P2VP) diblock copolymers. Briefly, commercially available PS-P2VP diblock copolymers were dissolved in toluene to form inverse micelles with the P2VP head groups constituting the micelle core. Sizeselected Pt NPs are created by dissolving $\mathrm{H}_{2} \mathrm{PtCl}_{6}$ into the polymeric solution. Subsequently, the nanocrystalline $\gamma$ - $\mathrm{Al}_{2} \mathrm{O}_{3}$ support (average size $\sim 40 \mathrm{~nm}$ ) is added. After drying, the encapsulating ligands are eliminated by annealing in $\mathrm{O}_{2}$ at $375{ }^{\circ} \mathrm{C}$ for $24 \mathrm{~h}$. Different NP shapes can be obtained by tuning the molecular weight of the different blocks of the encapsulating polymer, the metal/P2VP ratio (micelle loading), and the post preparation annealing treatment and atmosphere. ${ }^{20}$ Further details on the sample preparation method and synthesis parameters can be found in Refs. 20-22 and Table I. The NP diameters obtained by high-angle annular dark-field scanning transmission electron microscopy (TEM) are: $1.0 \pm 0.2 \mathrm{~nm}$ for $\mathrm{S} 1, \mathrm{~S} 2$, and $\mathrm{S} 4$, and $0.8 \pm 0.2 \mathrm{~nm}$ for $\mathrm{S} 3 .{ }^{20}$

\section{B. Structural characterization (EXAFS)}

EXAFS data were acquired at beamline X18B of the NSLS at Brookhaven National Laboratory in transmission mode using the Pt $L_{3}$ edge. The EXAFS samples were pre- 
TABLE I. Description of synthesis parameters and size (TEM) information of micellar Pt NPs supported on nanocrystalline $\gamma-\mathrm{Al}_{2} \mathrm{O}_{3}$. The TEM values correspond to NP diameters (D). The parameter " $L$ " represents the metal-salt to polymer head (P2VP) ratio used in the NP synthesis.

\begin{tabular}{lcccc}
\hline \hline \multicolumn{3}{c}{$\begin{array}{c}\text { Synthesis details of the } \\
\text { "as-prepared" Pt NPs } / \gamma-\mathrm{Al}_{2} \mathrm{O}_{3}\end{array}$} & \\
Sample & Polymer & $L$ & $\begin{array}{c}\text { Annealing } T \\
\left({ }^{\circ} \mathrm{C}\right)\left[\left(\% \mathrm{O}_{2}\right)\right]\end{array}$ & $\begin{array}{c}\text { TEM analysis after annealing } \\
+ \text { EXAFS measurements D }(\mathrm{nm})\end{array}$ \\
\cline { 2 - 4 } S1 & $\mathrm{PS}(16000)-\mathrm{P} 2 \mathrm{VP}(3500)$ & 0.05 & $375[50]$ & $1.0(2)$ \\
S2 & $\mathrm{PS}(16000)-\mathrm{P} 2 \mathrm{VP}(3500)$ & 0.1 & $425[70]$ & $1.0(2)$ \\
S3 & $\mathrm{PS}(27700)-\mathrm{P} 2 \operatorname{VP}(4300)$ & 0.1 & $375[50]$ & $0.8(2)$ \\
S4 & $\mathrm{PS}(27700)-\mathrm{P} 2 \mathrm{VP}(4300)$ & 0.2 & $375[50]$ & $1.0(2)$ \\
\hline \hline
\end{tabular}

pared by pressing the $\mathrm{Pt} / \gamma-\mathrm{Al}_{2} \mathrm{O}_{3}$ powders into thin pellets which were mounted in a sample cell that permitted sample heating via an external proportional-integral-derivative controller, liquid-nitrogen cooling, as well as the continuous flow of gases during data acquisition and online mass spectrometry analysis. A bulk $\mathrm{Pt}$ foil was measured simultaneously with all samples (in reference mode) for energy alignment and calibration purposes. Multiple scans (up to 6) were collected at each temperature of interest and averaged in order to improve the signal-to-noise ratio. Measurements were done at different temperatures under $\mathrm{H}_{2}\left(50 \% \mathrm{H}_{2}\right.$ balanced with $\mathrm{He}$ for a total flow rate of $50 \mathrm{ml} / \mathrm{min}$ ) or $\mathrm{He}$ (100\% He flow) atmospheres. Samples S1, S2, S4, and a Pt foil were measured in $\mathrm{H}_{2}$ while $\mathrm{S} 3$ was measured in $\mathrm{H}_{2}$ as well as in He.

Data processing and analysis with the IFEFFIT package $\mathrm{e}^{23}$ was conducted by analyzing the first shell Pt-Pt contribution for all temperature data concurrently, as described in Refs. 9 and 24, with the exception that the EXAFS Debye-Waller factors were obtained following the correlated Debye model (CDM) (Ref. 25) instead of the Einstein model. The CDM was used to extract the dynamic mean-square relative bondlength disorder, $\sigma_{\mathrm{d}}^{2}$, from the analysis of temperaturedependent EXAFS data, and through that $\theta_{D}$. The total bondlength disorder $\sigma^{2}$ also includes a configurational, or static, $\sigma_{\mathrm{s}}^{2}$ contribution: $\sigma^{2}=\sigma_{\mathrm{s}}^{2}+\sigma_{\mathrm{d}}^{2}$. Due to the linear dependence of the total disorder on temperature in the classical regime (i.e., when the temperature of the measurement is greater than the Einstein temperature, $\sim 190 \mathrm{~K}), \sigma_{\mathrm{s}}^{2}$ can be estimated either by extrapolating the total disorder function to zero temperature, or from the fit using the CDM (the present approach). The following parameters were varied in the EXAFS analysis: the corrections to the photoelectron energy origin, the nearest-neighbor bond lengths, the third cumulant of the first-NN (1NN) pair distribution function, and the values of $\theta_{D}$ and $\sigma_{\mathrm{S}}^{2}{ }^{24}$ We carefully investigated the effect of the energy origin $\left(E_{0}\right)$ on the values of the structural parameters (distances and third cumulants) extracted from the fits since they correlate. In most of the samples the x-ray absorption near-edge structure portion of the absorption coefficient exhibits a small shift (approximately 1-1.5 eV, depending on the sample) toward lower energies with increasing temperature. The same effect was observed earlier by Sanchez et al. ${ }^{9}$ To compensate the effect of this shift on the change in the phase of the EXAFS signals we compared two different fitting procedures: (1) the correction to the energy origin was varied independently for each temperature and (2) all the data were aligned in energy before extracting the EXAFS signals, and subsequently the energy origin correction, same for all data that were analyzed concurrently, was varied during the fitting routine. The best-fit results for these two approaches were very close (within the uncertainties). Supplementary Tables I and II (Ref. 26) contain details of the fitting parameters used for the analysis of EXAFS spectra acquired at different temperatures presented in our paper, in particular, the number of relevant independent data points, the number of variables, factors reflecting the fit quality (reduced $\chi^{2}$ and the $r$ factor), and values of the third cumulant.

Quantitative determination of the NP shape was carried out by analyzing low-temperature EXAFS data up to the fourth-NN (4NN) contribution, including multiple scattering, as described in Ref. 20, and references therein. Degeneracy of single- and multiple-scattering paths in EXAFS analysis is simply related to the coordination number of the nearest neighbors, and can be obtained from the nonlinear leastsquares fit of the theoretical EXAFS equation to the experimental data. ${ }^{23}$ Physically reasonable constraints between the fitting variables were applied to maximize the number of degrees of freedom in the fits. The passive electron reduction factor was fixed to that found for the Pt foil used as reference: 0.861 . Only the most important single- and collinear multiple-scattering paths were used in the fits through the fourth coordination shell. Among the multiple-scattering paths we used double- and triple-scattering paths to the $4 \mathrm{NN}$ through the intervening atom (first neighbor, or 1NN) along the same line. The total Debye-Waller values $\left(\sigma^{2}\right)$ for these paths were constrained to be the same as that of the $4 \mathrm{NN}$ $\mathrm{Pt}-\mathrm{Pt}$ pair. In addition, a double-scattering path connecting the central atom, its $1 \mathrm{NN}$, and another $1 \mathrm{NN}$ in the opposite direction was used in the fit. The disorder parameter of this path was varied independently from the others. Distance corrections were constrained to vary in accordance with the isotropic lattice expansion or contraction for all paths except for the first one, for which the third cumulant of the pair distribution function accounting for its asymmetry was varied as well. This approximation is justified by the apparent strong structural order in all particles, evident by comparing raw data in $r$ space for all the samples and the reference bulk $\mathrm{Pt}$ 
TABLE II. Model polyhedron shapes for fcc-Pt NPs providing the best fit to the experimental coordination numbers (EXAFS) and volume-weighted NP diameters (TEM). The volume-weighted TEM diameters (D), the total number of atoms in each NP $\left(N_{\mathrm{t}}\right)$, the ratio of the number of Pt atoms at the NP surface and perimeter to $N_{\mathrm{t}}\left(N_{\mathrm{s}} / N_{\mathrm{t}}\right)$, and the ratio of the number of Pt atoms in contact with the support to $N_{\mathrm{t}}\left(N_{\mathrm{c}} / N_{\mathrm{t}}\right)$ are given. A factor $(Q)$ representing the deviations of the coordination numbers and diameter of each of the model shapes with respect to the experimental samples are also included.

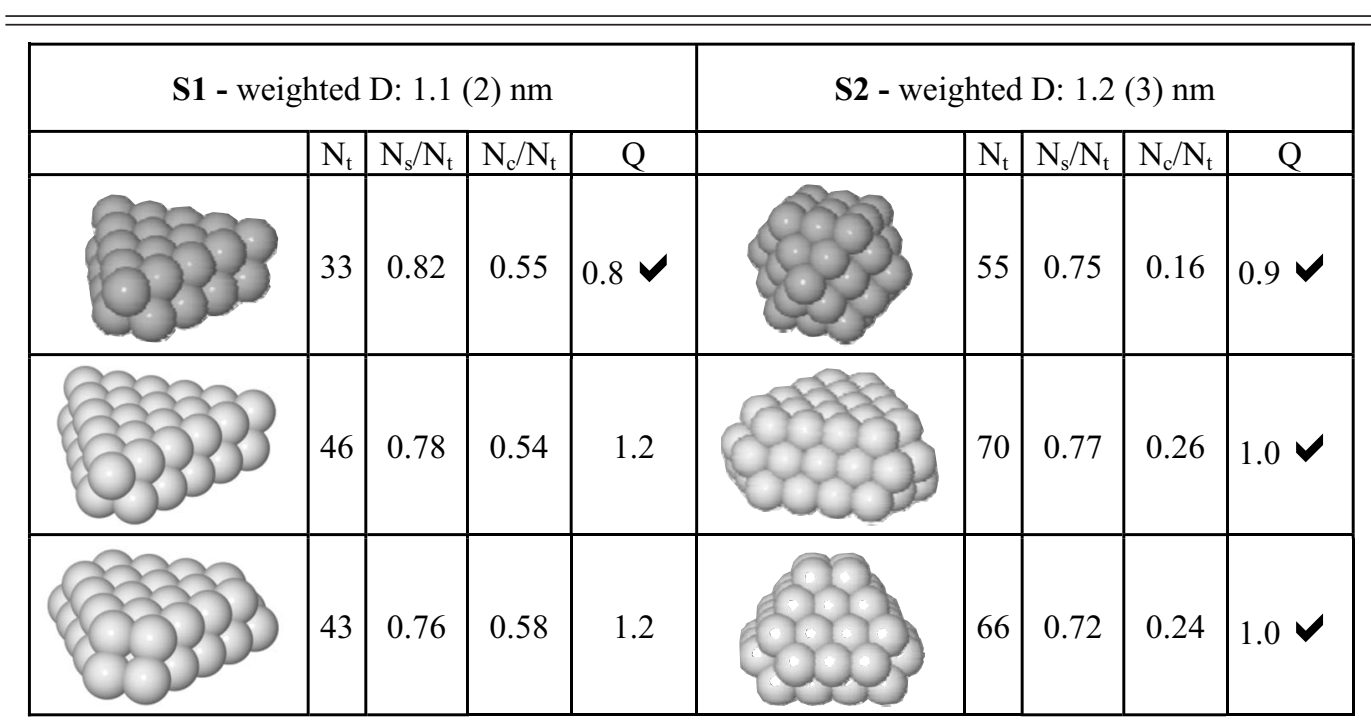

\begin{tabular}{|c|c|c|c|c|c|c|c|c|c|}
\hline \multicolumn{3}{|c|}{ S3 - weighted D: $0.9(2) \mathrm{nm}$} & \multicolumn{5}{c|}{ S4 - weighted D: 1.2 (2) nm } \\
\hline & $\mathrm{N}_{\mathrm{t}}$ & $\mathrm{N}_{\mathrm{s}} / \mathrm{N}_{\mathrm{t}}$ & $\mathrm{N}_{\mathrm{c}} / \mathrm{N}_{\mathrm{t}}$ & $\mathrm{Q}$ & & $\mathrm{N}_{\mathrm{t}}$ & $\mathrm{N}_{\mathrm{s}} / \mathrm{N}_{\mathrm{t}}$ & $\mathrm{N}_{\mathrm{c}} / \mathrm{N}_{\mathrm{t}}$ & $\mathrm{Q}$ \\
\hline & & & & & & & & & \\
\hline \\
\hline
\end{tabular}

foil [Fig. 5(b) of Ref. 20 and Fig. 1 of this work]. Coordination numbers of all single-scattering paths were varied independently; those of the multiple-scattering paths were constrained to those of the single-scattering paths to the $4 \mathrm{NN}$ or $1 \mathrm{NN}$, depending on the geometry of a particular path. Finally, the same energy correction $\left(E_{0}\right)$ was used for all paths. Supplementary Table III displays coordination numbers and distances of each coordination shell to the absorbing atom obtained from the multiple-scattering analysis of EXAFS data acquired for sample S3. ${ }^{26}$ The data were obtained at 173 $\mathrm{K}$ in $\mathrm{H}_{2}$ after NP reduction. Reference 20 contains analogous information for the rest of the samples discussed in the present paper.

The possibility of morphological changes in our samples as a function of temperature has not been accounted for in our analysis. This approach decreases the number of fitting parameters and is justified by the following arguments: (i) the behavior of our raw EXAFS data throughout the entire temperature range is not consistent with a size or shape change, (ii) our samples were measured under a hydrogen atmosphere that passivates the NPs and stabilizes their shapes, and (iii) the analysis of the vibrational properties of our NPs was conducted up to a maximum annealing temperature of $375{ }^{\circ} \mathrm{C}$, which is the same temperature used for sample calcination (for $24 \mathrm{~h}$ ) prior to the EXAFS measurements. If any changes in the NP morphology (size and/or shape) were to occur at $375{ }^{\circ} \mathrm{C}$, they should have already taken place before the EXAFS measurements. Evidence of the lack of NP sintering at this annealing temperature can be 

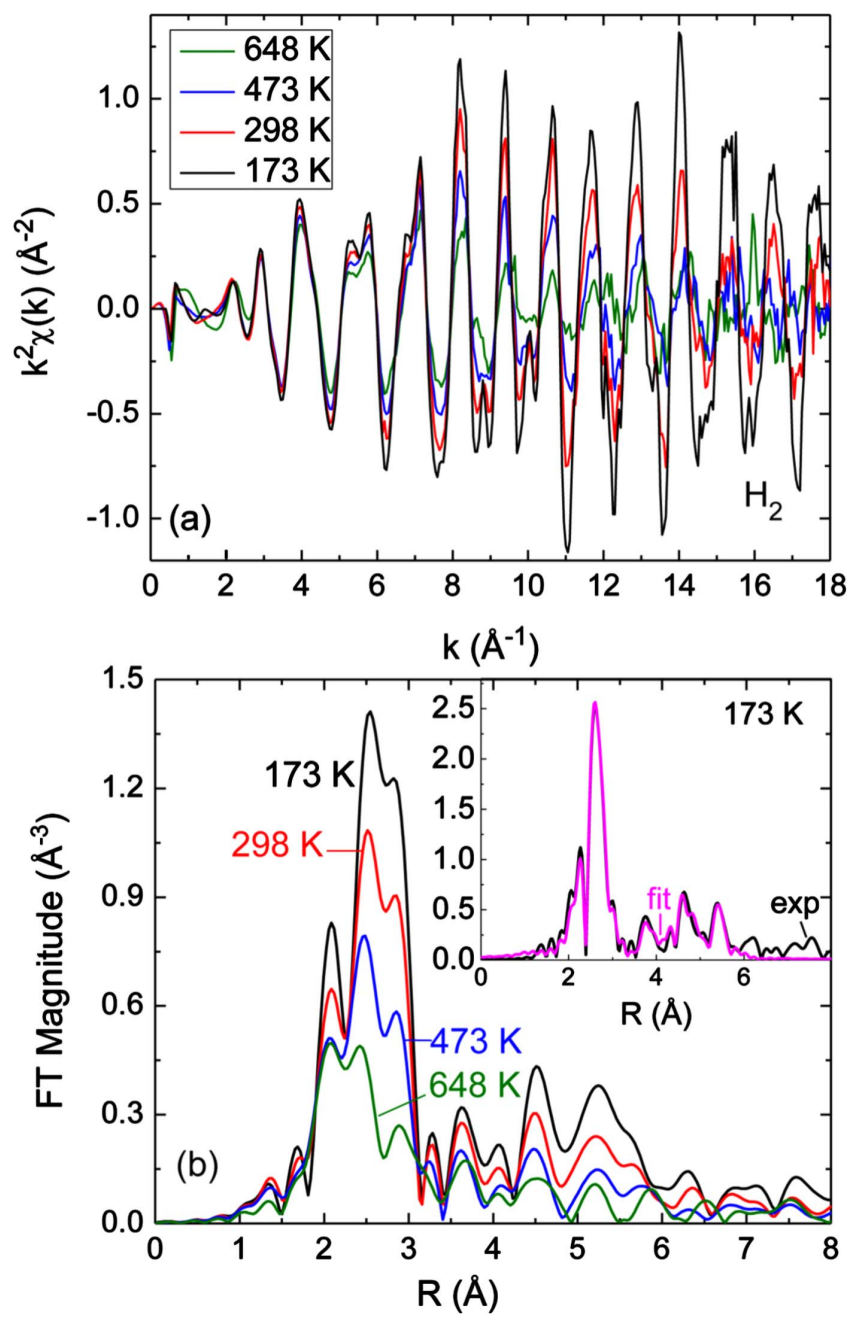

FIG. 1. (Color online) Temperature-dependent EXAFS data in (a) $k$ space $\left(k^{2}\right.$-weighted) and (b) $r$ space of $\mathrm{S} 3$ measured in $\mathrm{H}_{2}$ atmosphere. The inset displays the experimental data acquired at $173 \mathrm{~K}$ together with the corresponding multiple-scattering fit.

found in the TEM images of analogous samples depicted in Ref. 20.

\section{Nanoparticle shape modeling}

The shapes of our Pt NPs have been resolved by matching structural information obtained experimentally via EXAFS (coordination numbers up to the fourth-nearest neighbor, $N_{1}-N_{4}$ ) and TEM (NP diameter, $D$ ) to analogous data extracted from a database containing $\sim 4000$ model fcc NP shapes. Only closed shell clusters were considered in the models. In our analysis, after taking into consideration the error bars in the EXAFS coordination numbers as well as the TEM diameters, we have found on average only three-model fcc cluster shapes consistent with all five experimental parameters $\left(N_{1}, N_{2}, N_{3}, N_{4}\right.$, and $\left.D\right)$. Table II shows the best three-model shapes for each of the samples investigated together with information on the total number of atoms, the ratio of the number of Pt atoms at the NP surface, and perimeter to $N_{\mathrm{t}}\left(N_{\mathrm{s}} / N_{\mathrm{t}}\right)$, the ratio of the number of Pt atoms in contact with the support to $N_{\mathrm{t}}\left(N_{\mathrm{c}} / N_{\mathrm{t}}\right)$, and a factor $(Q)$ in- dicating how much each of the specific model shapes deviates from the experimentally measured EXAFS coordination numbers and volume-weighted TEM NP diameters. The shapes with the best $Q$ factors are depicted in a darker color in Table II and appear as inset in Fig. 3. The $Q$ factor has been obtained following Eq. (1):

$$
Q=\sqrt{\frac{N E_{1}^{2}+N E_{2}^{2}+N E_{3}^{2}+D E^{2}}{4}},
$$

where $N E_{1}, N E_{2}$, and $N E_{3}$ are normalized errors of the experimental first, second, and third coordination numbers, and $D E$ is the normalized error of the TEM diameter. The latter errors are calculated following Eqs. (2) and (3):

$$
\begin{gathered}
N E=\frac{\text { Experimental } C N-\text { Model } C N}{\text { Experimental error of } C N}, \\
D E=\frac{\text { Experimental } D-\text { Model } D}{\text { Experimental error of } D},
\end{gathered}
$$

where $D$ is the NP diameter and $C N$ are the respective coordination numbers $\left(N_{1}, N_{2}\right.$, or $\left.N_{3}\right)$. In the calculation of the $Q$ factor we have not considered the fourth coordination number extracted from EXAFS. As shown by Jentys, ${ }^{27}$ either $N_{3}$ or $N_{4}$ can be used interchangeably to estimate the particle shape, but since $N_{3}$ is generally larger than $N_{4}$ for most shapes of fcc particles (e.g., 24 vs 12 in the bulk), the former number has greater impact on the shape determination.

A low- $Q$ factor indicates a good fit between experimental and model $C N$ and $D$. As can be seen in the Table II, only one shape provides a $Q$ factor $\leq 1$ for samples $\mathrm{S} 1$ and $\mathrm{S} 3$, while as few as three shapes satisfy that constraint for $\mathrm{S} 2$ and S4, indicating the low degeneracy in the determination of the cluster shape for samples containing NPs in our size range $(\sim 0.8-1 \mathrm{~nm})$. For $\mathrm{S} 4$, two shapes with identical $Q$ factor were obtained. The final shape selected to be displayed as inset in Fig. 3 is a shape (85-atom NP) that we have also experimentally resolved on identically prepared Pt NPs supported on $\mathrm{TiO}_{2}(110)$ via scanning tunneling microscopy (STM, not shown here). For the rest of the samples, all shapes characterized by the lowest $Q$ factors in Table II have also been observed by our group via STM on analogous micellar Pt NPs. Examples of the NP shapes resolved by STM can be found in Refs. 20 and 28.

\section{RESULTS}

EXAFS spectra in (a) $k$ space and (b) $r$ space from a ligand-free micellar Pt NP sample supported on $\gamma-\mathrm{Al}_{2} \mathrm{O}_{3}$ (S3) acquired in $\mathrm{H}_{2}$ at temperatures ranging from 173 to 648 $\mathrm{K}$ are shown in Fig. 1. A decrease in the intensity of the EXAFS signals with increasing temperature is evident. Such effect can be attributed to an increase in the bond-length disorder in the framework of $\mathrm{Pt}$ atoms, to a decrease in the Pt-Pt first-nearest-neighbor coordination number due to changes in the NP size and/or shape, or to both. We can single out the disorder as the dominant factor responsible for this behavior when we examine the $k$-space data, Fig. 1(a). If 
TABLE III. First-nearest-neighbor coordination number $\left(N_{1}\right)$, thermal expansion coefficient $(\alpha)$, static disorder $\left(\sigma_{\mathrm{s}}^{2}\right)$, and Debye temperature $\left(\theta_{D}\right)$ of samples S1-S4 and of a bulk-like Pt foil. All samples were measured in $\mathrm{H}_{2}$. S3 was also measured in He. For the model polyhedron shapes, the total number of atoms in each NP $\left(N_{\mathrm{t}}\right)$, the ratio of the number of Pt atoms at the NP surface and perimeter to $N_{\mathrm{t}}\left(N_{\mathrm{s}} / N_{\mathrm{t}}\right)$, and the ratio of the number of Pt atoms in contact with the support to $N_{\mathrm{t}}\left(N_{\mathrm{c}} / N_{\mathrm{t}}\right)$ are also given. Details on the NP shape selection can be found in Table II. The $N_{\mathrm{s}} / N_{\mathrm{t}}$ and $N_{\mathrm{c}} / N_{\mathrm{t}}$ ratios of samples $\mathrm{S} 2$ and S4 correspond to the average of three shapes that best fitted the experimental coordination numbers and NP diameter. Only one shape was observed to fulfill the same criteria for samples S1 and S3. The largest facets within each NP were selected as facets in contact with the support: (111) for S1, S3, and S4, and (100) for S2.

\begin{tabular}{lccccccc}
\hline \hline Sample & $N_{1}$ & $\begin{array}{c}\alpha \\
\left(10^{-6} \mathrm{~K}^{-1}\right)\end{array}$ & $\begin{array}{c}\sigma_{\mathrm{s}}^{2} \\
\left(\AA^{2}\right)\end{array}$ & $\begin{array}{c}\theta_{D} \\
(\mathrm{~K})\end{array}$ & $N_{\mathrm{t}}$ & $N_{\mathrm{s}} / N_{\mathrm{t}}$ & $N_{\mathrm{c}} / N_{\mathrm{t}}$ \\
\hline $\mathrm{S} 1\left(\mathrm{H}_{2}\right)$ & $7.5(3)$ & $-2(3)$ & $0.0028(3)$ & $275(9)$ & 33 & 0.82 & 0.55 \\
$\mathrm{~S} 2\left(\mathrm{H}_{2}\right)$ & $9.0(3)$ & $3(2)$ & $0.0019(2)$ & $284(7)$ & 55 & $0.75(3)$ & $0.22(3)$ \\
$\mathrm{S} 3\left(\mathrm{H}_{2}\right)$ & $8.0(2)$ & $-3(4)$ & $0.0013(2)$ & $250(5)$ & 44 & 0.84 & 0.23 \\
$\mathrm{~S} 3(\mathrm{He})$ & $7.8(3)$ & $-15(4)$ & $0.0011(2)$ & $240(6)$ & & & \\
$\mathrm{S} 4\left(\mathrm{H}_{2}\right)$ & $8.6(2)$ & $3(2)$ & $0.0010(3)$ & $262(6)$ & 85 & $0.74(2)$ & $0.15(3)$ \\
Pt bulk & 12 & $11(1)$ & $0.00017(8)$ & $244(3)$ & & & \\
\hline \hline
\end{tabular}

the NP size and/or shape were changing with temperature, the coordination number effect would be visible for all $k$ values, since it affects the EXAFS signal as a constant multiplicative factor. However, the decrease in the intensity of our $k$-space data is more prominent at the end of the $k$ range than at the beginning. Such observation is in agreement with the disorder effect since it is expected to increase with increasing $k$.

A representative multiple-scattering fit of the $173 \mathrm{~K}$ spectrum is included as inset in Fig. 1. Supplementary Figs. 1 and 2 show EXAFS data for the remaining samples at all temperatures in $k$ space, and representative fits in $r$ space to temperature-dependent data from S2. ${ }^{26}$ Supplementary Fig. 3 displays the imaginary part of the $k^{2}$-weighted EXAFS data, fit, and residual contribution obtained for S2 and S3 at 173$183 \mathrm{~K}^{26}$

Figure 2 displays (a) Pt-Pt distances $(R)$ and (b) the dynamic bond-length disorders $\left(\sigma_{\mathrm{d}}^{2}\right)$ in the temperature range of 150-700 K for samples S1-S4 and for a reference bulk like Pt foil. All measurements were carried out under a continuous $\mathrm{H}_{2}$ flow, with the exception of sample $\mathrm{S} 3$, for which a $\mathrm{He}$ and $\mathrm{H}_{2}$ adsorbate effect was clearly observed [inset, Fig. 2(a)]. The data in Fig. 2(a) correspond to the best-fit results of the experimental EXAFS spectra obtained for the 1NN Pt-Pt bond lengths $(R)$ at different temperatures.

The dynamic bond-length disorders $\left(\sigma_{\mathrm{d}}^{2}\right)$ obtained from the fits of the experimental EXAFS data following the CDM (Ref. 25) are shown in Fig. 2(b). In the high-temperature approximation $\sigma_{\mathrm{d}}^{2}=k_{\mathrm{B}} T / k \sim T / \theta_{D}^{2}$, with $k$ being the effective force constant. Hence, the slope of the temperature dependence of $\sigma_{\mathrm{d}}^{2}$ depends on $\theta_{D}$, with larger Debye temperature giving smaller slopes. The data in Fig. 2(b) reveal a striking behavior of our NPs in this regard, which will be discussed in greater detail below. The best-fit values of $\sigma_{\mathrm{s}}^{2}$ are shown in Table III together with the first-nearest-neighbor coordination numbers $\left(N_{1}\right)$ and $\theta_{D}$.

Figure 3 displays the Debye temperatures obtained from the CDM analysis of our EXAFS data [Fig. 2(b)] as a function of $N_{1}$. Samples S1, S2, and S4 have the same TEM diameter $(1.0 \pm 0.2 \mathrm{~nm})$ but different shapes. The data correspond to samples passivated by $\mathrm{H}_{2}$ under identical conditions.

\section{DISCUSSION}

The thermal-expansion coefficients $(\alpha)$ of the Pt NP samples were estimated from the slope of the linear interpolation of the data in Fig. 2(a), Table III. Changes in the magnitude and sign of $\alpha$ are observed when comparing the different samples. As expected, the Pt foil displays a positive thermal expansion. Surprisingly, only two of our samples (S2 and S4) follow the same trend, although a smaller $\alpha$ than for bulk Pt is obtained, suggesting NP stiffening. The remaining samples (S1 and S3) show negative thermal expansion with a more prominent effect observed for the sample containing the NPs with the smallest TEM diameter $(\sim 0.8 \mathrm{~nm}, \mathrm{~S} 3)$. The negative slope of the $R$ vs $(T)$ curves in supported Pt NPs represents markedly nonbulk behavior, which can be attributed to the competing interactions of cluster-support and cluster-adsorbate $\left(\mathrm{H}_{2}\right.$ or $\mathrm{He}$ in our case $)$ effects, both depending on the NP size and shape. The effect of $\mathrm{H}$ adsorbates on the Pt-Pt spacing is expected to cause relief of both the surface tension and compressive stress which is always present in NPs, and thus increase their average bond lengths. We postulate that the extent of adsorbate-induced relief can be correlated with the surface-to-volume ratios of different NPs. This effect is demonstrated in the inset of Fig. 2(a), where larger $R$ distances were measured for $\mathrm{S} 3$ under $\mathrm{H}_{2}$ as compared to He. A more prominent negative thermal expansion was observed for S3 under He. Sample S3 has the largest surface-to-volume ratio (Table III) among all samples and consequently is expected to be most affected by the presence of adsorbates. An alternative mechanism proposed to explain negative thermal expansion for materials characterized by strong bonding anisotropy relates to enhanced atomic displacements in the transverse direction to the bond. ${ }^{29}$

Analysis of the EXAFS data in Fig. 2(b) obtained for the Pt foil via the CDM lead to a $\theta_{D}$ of $244 \pm 3 \mathrm{~K}$, which is in 


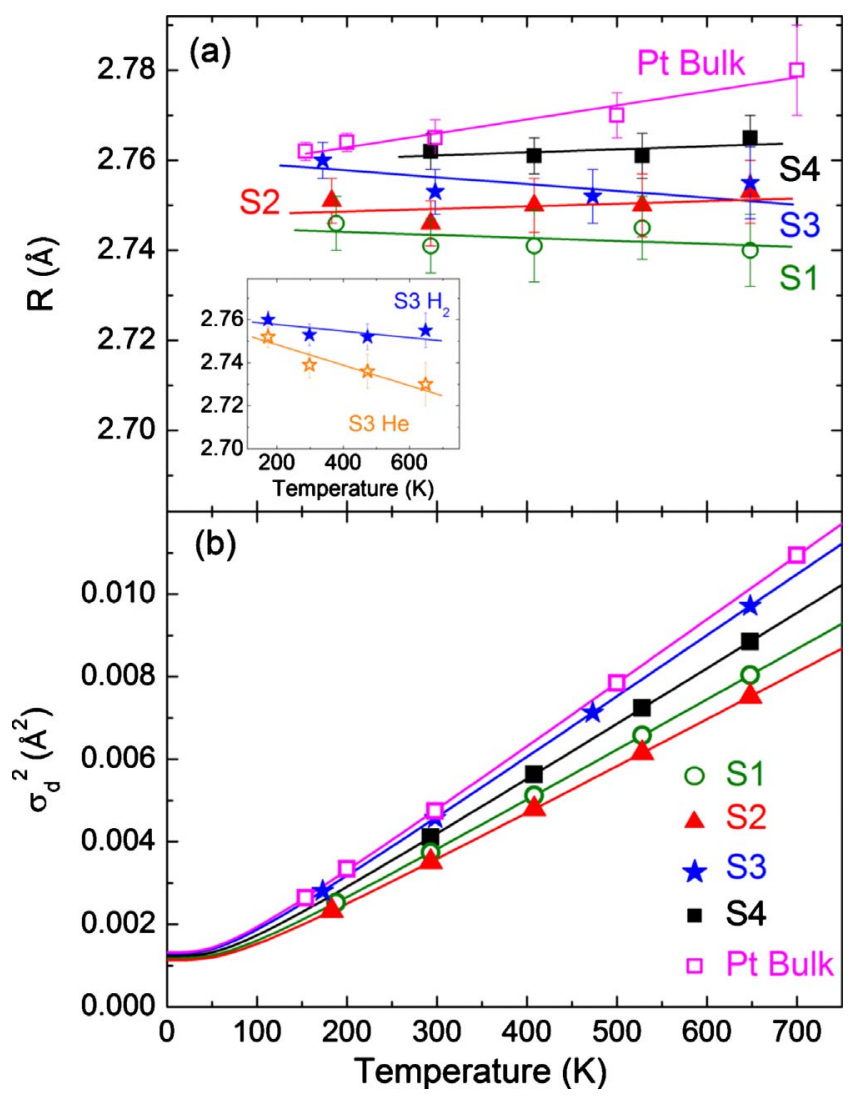

FIG. 2. (Color online) (a) $T$-dependent Pt-Pt bond length $(R)$ obtained from EXAFS measurements on samples S1-S4 and on a bulk Pt foil. All samples were measured in $\mathrm{H}_{2}$. The inset in (a) displays Pt-Pt distances from S3 measured in $\mathrm{He}$ or $\mathrm{H}_{2}$. The solid lines represent linear fits to the experimental data. (b) Dynamic contribution $\left(\sigma_{\mathrm{d}}^{2}\right)$ to the total $\sigma^{2}$ obtained under $\mathrm{H}_{2}$ flow and analyzed with the correlated Debye model (solid lines). Symbols correspond to the temperatures at which the EXAFS data were measured.

agreement with literature reports. ${ }^{19}$ Surprisingly, significantly higher Debye temperatures (262-284 K) were observed for samples S1, S2, and S4 ( $\sim 1 \mathrm{~nm}$ in diameter). However, a similar $\theta_{D}$ value to that of the bulk Pt was obtained for the sample with the smallest NPs $\left(\mathrm{S} 3, \sim 0.8 \mathrm{~nm}, \theta_{D}\right.$ $=250 \pm 5 \mathrm{~K}$ ). If we compare the Pt-Pt bond lengths in Fig. 2(a) for different NP samples at the lowest temperatures, i.e., when the hydrogen coverages, and hence the ordering of NP facets, are the greatest, ${ }^{30,31}$ we can see that samples S1 and $\mathrm{S} 2$, which are characterized by the highest $\theta_{D}$ (Table III and Fig. 3), have the smallest values of Pt-Pt bond lengths. This suggests some correlation between increased compressive strains and enhanced $\theta_{D}$. In fact, due to the asymmetry of the effective pair potential, where the short distance branch is steeper than the long distance branch (due to the hard-core repulsion), the Pt-Pt bond stiffness should be large in the systems having bonds with large compressive strain, namely $\mathrm{S} 1$ and S2. It should also be mentioned that the compressive strain present in supported NP systems might have different origins as, for example, the presence of ligands on the NP surface (not the present case), adsorbates, or a support $\left(\gamma-\mathrm{Al}_{2} \mathrm{O}_{3}\right)$. However, the explanation of the enhanced $\theta_{D}$ ob-

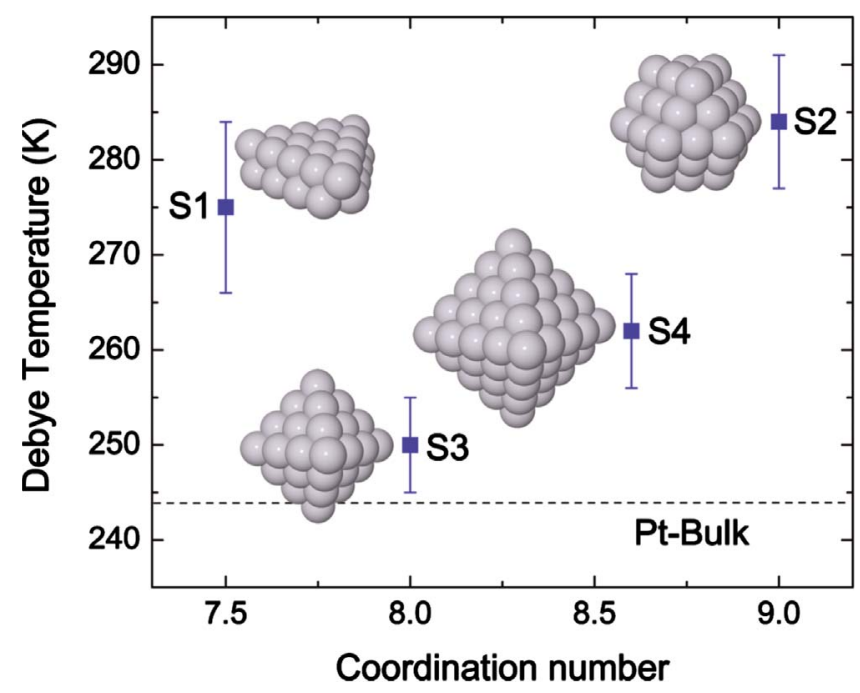

FIG. 3. (Color online) Debye temperature of $\gamma-\mathrm{Al}_{2} \mathrm{O}_{3}$ supported Pt NPs as a function of the first-nearest-neighbor coordination number $\left(N_{1}\right)$. All samples were measured in $\mathrm{H}_{2}$. The insets show corresponding model fcc-Pt NP shapes (Ref. 20).

served appears to be more complex than a direct one-to-one correlation with a contraction of Pt-Pt distances, $R$. Such contraction is theoretically predicted for free (unsupported) clusters due to surface tension arguments while the same systems show $\theta_{D}$ smaller than the corresponding bulk metals. ${ }^{1}$ In fact, based on EXAFS measurements, Yokoyama et al. ${ }^{18}$ reported metal-metal bond contractions for small $\mathrm{Ag}$ and Pd NPs (1.3-1.5 nm) accompanied by reduced $\theta_{D}$ with respect to the bulk. Also, the above explanation for the enhanced $\theta_{D}$ values in terms of a contraction of $R$ does not take into account the distinct differences (outside of the error bars) observed in $\theta_{D}$ for the three samples (S1, S2, and S4) characterized by the same TEM diameter $(\sim 1.0 \mathrm{~nm})$.

In order to gain further insight into the origin of the anomalous behavior of the Debye temperature of our NP samples we should consider their different geometrical structures. As was described in detail in the Results section, the shapes of our Pt NPs have been resolved by matching structural information obtained experimentally via EXAFS $\left(N_{1}\right.$, $N_{2}, N_{3}$ ) and TEM (NP diameter, $D$, obtained from Z-contrast images) to analogous data extracted from a database containing model fcc NP shapes. ${ }^{20}$ The most representative shapes for each of the samples are a truncated octahedron (S1), cuboctahedron (S2), and octahedron (S3 and S4). As can be seen in Fig. 3, all samples but sample S1 contain Pt NPs with a three-dimensional (3D) shape. This conclusion is in agreement with the static disorder values displayed in Table III, since the largest $\sigma_{\mathrm{s}}^{2}$ was obtained for S1 $\left(\sim 0.0028 \AA^{2}\right)$, which according to our model-shape analysis is the sample with the highest contact area with the support [twodimensional (2D) shape]. The low $\sigma_{\mathrm{s}}^{2}$ value of $\sim 0.0010 \AA^{2}$ obtained for our smallest NPs (S3) is consistent with their 3D shape, with a relatively large surface area available for hydrogen adsorption, leading to relaxation and NP faceting.

If one compares S3 and S4, with identical octahedron shapes but different sizes, we can see that the smallest NPs (S3) displayed also the smallest Debye temperature. We at- 
tribute this to two concomitant effects: (i) softening of interatomic force constants due to the presence of a larger relative number of loosely bound surface atoms in S3 than in S4 (see the $N_{\mathrm{s}} / N_{\mathrm{t}}$ ratio values in Table III) and (ii) the possibly lower degree of long-range atomic ordering expected for the smaller NPs since even for bulk systems reduced $\theta_{D}$ were observed for amorphous versus crystalline materials. ${ }^{13}$

Interestingly, irrespective of the particular cluster size and shape, a correlation between $\theta_{D}$ and $N_{1}$ was observed for all NP samples with 3D shape (S2, S3, and S4), Fig. 3. More specifically, a reduction in $\theta_{D}$ was observed with decreasing $N_{1}$ or increasing number of missing bonds (with respect to $N_{1}=12$ for bulk fcc-Pt). An exception to this trend is the sample containing 2D NPs (S1). As can be seen in Table III, the latter NPs have the highest contact area with the support $\left(N_{\mathrm{c}} / N_{\mathrm{t}}=0.55\right)$, a factor that opposes the influence of undercoordinated atoms in these NPs since it leads to increased compressive strain and higher $\theta_{D}$. For the rest of the samples, the relative number of atoms in contact with the support is nearly constant $(0.15-0.23)$, leading to a comparable support influence on $\theta_{D}$, and allowing us to separate size/shape effects from support effects. Furthermore, our data also indicate that adsorbate effects play only a secondary role in determining the final magnitude of $\theta_{D} \cdot \mathrm{H}_{2}$ is known to release stress [see inset in Fig. 2(a)], an effect that counterbalances the support-induced stiffening and leads to a reduction in $\theta_{D}$. Interestingly, even though the relative fraction of atoms at the NP surface is nearly identical for $\mathrm{S} 1$ and $\mathrm{S} 3\left(N_{\mathrm{s}} / N_{\mathrm{t}}\right.$ ratios in Table III), the higher $\theta_{D}$ obtained for $\mathrm{S} 1$ as compared to S3 is attributed to the dominating support effect, since a much larger fraction of $\mathrm{Pt}$ atoms are in contact with the support in S1 (see Table III).

\section{CONCLUSIONS}

Our study reveals an overall significant shortening of the Pt-Pt bond lengths and a drastic increase in the Debye temperatures of ligand-free, size-, and shape-selected Pt NPs synthesized by inverse micelle encapsulation and supported on nanocrystalline $\gamma-\mathrm{Al}_{2} \mathrm{O}_{3}$. We attribute the origin of the enhanced $\theta_{D}$ of our NPs as compared to bulk Pt to compressive stress from the NP/support interface as well as to inhomogeneous strain caused by competing contributions from atoms at the NP surface (leading to relaxation and reduced $\theta_{D}$ ) and in the interior of the NPs (experiencing enhanced stiffening leading to increased $\theta_{D}$ ).

In this work we have demonstrated that the lattice dynamics and thermal behavior of NPs is affected by their geometric properties. Our experimental data provide important validation for nanothermodynamics theories that, as we have shown qualitatively in this work, should incorporate geometrical effects (size and shape) and environmental interactions (e.g., adsorbate and support) to adequately describe thermal properties at the nanoscale.

\section{ACKNOWLEDGMENTS}

The authors are grateful for the beamline support of N. Marinkovic, electron microscopy measurements by L. Li and J. C. Yang, and funding from the U.S. National Science Foundation (Grants No. NSF-DMR-1006232 and No. NSFDMR-0906562). Synchrotron Catalysis Consortium facilities at NSLS where the X-ray absorption measurements were conducted are supported by the U.S. Department of Energy (Grant No. DE-FG02-05ER15688). NSLS is supported by the U.S. Department of Energy (Grant No. DE-AC0298CH10866).

\footnotetext{
*Corresponding author.

†roldan@physics.ucf.edu

†anatoly.frenkel@yu.edu

${ }^{1}$ H. Yildirim, A. Kara, and T. S. Rahman, J. Phys.: Condens. Matter 21, 084220 (2009).

${ }^{2}$ A. Kara and T. S. Rahman, Phys. Rev. Lett. 81, 1453 (1998).

${ }^{3}$ B. Roldan Cuenya, A. Naitabdi, J. Croy, W. Sturhahn, J. Y. Zhao, E. E. Alp, R. Meyer, D. Sudfeld, E. Schuster, and W. Keune, Phys. Rev. B 76, 195422 (2007).

${ }^{4}$ B. Roldan Cuenya, W. Keune, R. Peters, E. Schuster, B. Sahoo, U. von Hörsten, W. Sturhahn, J. Zhao, T. S. Toellner, E. E. Alp, and S. D. Bader, Phys. Rev. B 77, 165410 (2008).

${ }^{5}$ B. Roldan Cuenya, L. K. Ono, J. R. Croy, A. Naitabdi, H. Heinrich, J. Zhao, E. E. Alp, W. Sturhahn, and W. Keune, Appl. Phys. Lett. 95, 143103 (2009).

${ }^{6}$ B. Fultz, Prog. Mater. Sci. 55, 247 (2010).

${ }^{7}$ T. L. Hill, J. Chem. Phys. 36, 3182 (1962).

${ }^{8}$ J. H. Kang, L. D. Menard, R. G. Nuzzo, and A. I. Frenkel, J. Am. Chem. Soc. 128, 12068 (2006).

${ }^{9}$ S. I. Sanchez, L. D. Menard, A. Bram, J. H. Kang, M. W. Small, R. G. Nuzzo, and A. I. Frenkel, J. Am. Chem. Soc. 131, 7040 (2009).

${ }^{10}$ F. D. Vila, J. J. Rehr, J. Kas, R. G. Nuzzo, and A. I. Frenkel,
}

Phys. Rev. B 78, 121404 (2008).

${ }^{11}$ R. Giulian, L. L. Araujo, P. Kluth, D. J. Sprouster, C. S. Schnohr, G. J. Foran, and M. C. Ridway, J. Phys.: Condens. Matter 21, 155302 (2009)

${ }^{12}$ M. Dubiel, J. Haug, H. Kruth, H. Hofmeister, and W. Seifert, J. Phys.: Conf. Ser. 190, 012123 (2009).

${ }^{13}$ L. L. Araujo, P. Kluth, G. de M. Azevedo, and M. C. Ridgway, Phys. Rev. B 74, 184102 (2006).

${ }^{14}$ Q. F. Gu, G. Krauss, W. Steurer, F. Gramm, and A. Cervellino,Phys. Rev. Lett. 100, 045502 (2008).

${ }^{15}$ B. Gilbert, F. Huang, H. Zhang, G. A. Waychunas, and J. F. Banfield, Science 305, 651 (2004).

${ }^{16}$ J. Rockenberger, L. Troger, A. L. Rogach, M. Tischer, M. Grundmann, A. Eychmuller, and H. Weller, J. Chem. Phys. 108, 7807 (1998).

${ }^{17}$ T. Comaschi, A. Balerna, and S. Mobilio, Phys. Rev. B 77, 075432 (2008).

${ }^{18}$ T. Yokoyama, S. Kimoto, and T. Ohta, Jpn. J. Appl. Phys. 28, L851 (1989).

${ }^{19}$ L. J. Giovanetti, J. M. Ramallo-Lopez, F. G. Requejo, D. I. Garcia-Gutierrez, M. J. Yakaman, and A. F. Craievich, J. Phys. Chem. C 111, 7599 (2007).

${ }^{20}$ B. Roldan Cuenya, J. R. Croy, S. Mostafa, F. Behafarid, L. Li, 
Z. Zhang, J. C. Yang, Q. Wang, and A. I. Frenkel, J. Am. Chem. Soc. 132, 8747 (2010).

${ }^{21}$ J. R. Croy, S. Mostafa, H. Heinrich, and B. Roldan Cuenya, Catal. Lett. 131, 21 (2009).

${ }^{22}$ A. Naitabdi, F. Behafarid, and B. Roldan Cuenya, Appl. Phys. Lett. 94, 083102 (2009).

${ }^{23}$ M. Newville, J. Synchrotron Radiat. 8, 322 (2001).

${ }^{24}$ A. I. Frenkel, C. W. Hills, and R. G. Nuzzo, J. Phys. Chem. B 105, 12689 (2001).

${ }^{25}$ E. Sevillano, H. Meuth, and J. J. Rehr, Phys. Rev. B 20, 4908 (1979).

${ }^{26}$ See supplementary material at http://link.aps.org/supplemental/ 10.1103/PhysRevB.82.155450 for further details on the EXAFS parameters used for the fit of the experimental EXAFS data, best fit results, fit quality, as well as additional EXAFS spectra in $k$ and $r$-space and corresponding fits.

${ }^{27}$ A. Jentys, Phys. Chem. Chem. Phys. 1, 4059 (1999).

${ }^{28}$ S. Mostafa, F. Behafarid, J. R. Croy, L. K. Ono, L. Li, J. C. Yang, A. I. Frenkel, and B. Roldan Cuenya, J. Am. Chem. Soc.(to be published).

${ }^{29}$ S. I. Ahmed, G. Dalba, P. Fornasini, M. Vaccari, F. Rocca, A. Sanson, J. Li, and A. W. Sleight, Phys. Rev. B 79, 104302 (2009).

${ }^{30}$ L. L. Wang and D. D. Johnson, J. Am. Chem. Soc. 129, 3658 (2007).

${ }^{31}$ S. Giorgio, M. Cabié, and C. R. Henry, Gold Bull. 41, 167 (2008). 